\title{
Virtual Reality Simulation Training for Ebola Deployment
}

Luca Ragazzoni, MD; Pier Luigi Ingrassia, MD, PhD; Lina Echeverri, MD; Fabio Maccapani, MD; Lizzy Berryman; Frederick M. Burkle, Jr, MD, MPH, DTM; Francesco Della Corte, MD

\section{ABSTRACT}

Both virtual and hybrid simulation training offer a realistic and effective educational framework and opportunity to provide virtual exposure to operational public health skills that are essential for infection control and Ebola treatment management. This training is designed to increase staff safety and create a safe and realistic environment where trainees can gain essential basic and advanced skills. (Disaster Med Public Health Preparedness. 2015;9:543-546)

Keywords: Ebola, professionalization, simulation, education and training

A s a World Health Organization (WHO) Level 3 Public Health Emergency of International Concern, the Ebola epidemic requires an unprecedented coordinated international response to contain and control the lethal virus. This response includes effective pre-deployment education and training of an expatriate health care workforce called to fill the requirements of foreign medical teams. Clinicians feel uneasy about treating a highly transmissible infection for which there is no vaccine, for which there is no specific therapy, and which is highly lethal. Unwillingness to respond to infectious emergencies, such as pandemic influenza, is already well documented. In this regard, preevent preparation and training plays a determinant role. ${ }^{1}$ Unfortunately, secondary infections among indigenous and expatriate health care workers is extremely high, which highlights the inadequate infection control practices and protections in many treatment centers. ${ }^{2}$ Being prepared requires the attainment of several operationallevel public health skills that are beyond the experience and knowledge base of most practitioners not familiar with public health emergencies arising from infectious disease outbreaks. This suggests that a concerted effort must be made to reaching a level of operational public health professionalization through more effective education and training. ${ }^{3}$ Unfortunately, such training is expensive and may be unrealistic in content and experience outside the affected country. If such hurdles are overcome, it is suggested that there would be a greater willingness of health care workers to engage in direct care delivery.

\section{CURRENT TRAINING INITIATIVES}

For obvious reasons, the international medical community is very sensitive to the development of effective pre-deployment training programs to ensure the adequate safety of humanitarian workers. WHO and Médecins Sans Frontières (MSF) have worked to create standard training center units and to multiply these units across the world to enlarge the number of workers equipped through advanced knowledge and operational skills. To understand the working principles and protocols currently used in a generic Ebola treatment center (ETC) or Ebola treatment unit (ETU), these organizations have built mock ETCs and ETUs to better expose humanitarian workers to a realistic environment. Scheduled single training courses are designed to improve technical skills in the application of personal protective equipment (PPE) in unfamiliar settings. However, these courses are challenging to organize, standardize, and fund and may be disruptive to already compromised clinical settings. Moreover, little is known about the assessment measures presently used during these training sessions, because there is no validated way to measure the humanitarian workers' learning process or operational understanding of specific treatment protocols. A rigorous evaluation methodology designed to detect potential errors and to verify the technical skills acquired by the participant (e.g., ability to apply and remove the PPE) is crucial for both the safety and confidence of humanitarian workers.

\section{VIRTUAL REALITY SIMULATION}

Simulation training is a recognized efficient and effective cornerstone of modern medical education and training, with a strong potential for generating positive learning outcomes. ${ }^{4}$ With increasing frequency, simulation-based medical education is being used for formative and summative evaluations 
including showing the efficacy of live simulation in the assessment of technical skills after a disaster medicine training session. ${ }^{5}$ Moreover, virtual reality (VR) simulation has been shown to be a valid, clinically appropriate, and cost-effective training method by achieving a quality degree of projected realism. ${ }^{5}$ As such, VR simulation has uniquely allowed health care providers and humanitarian workers to train for various emergency and disaster scenarios. Currently, the Research Center in Emergency and Disaster Medicine and Computer Science applied to Medical Practice (CRIMEDIM) is in partnership with Save the Children International (SCI) and their Ebola response team in replicating one of SCI's ETCs. Staff are walked through a VR version of the ETC, which allows them to familiarize themselves with the high-risk environment and to practice complex protocols "in place" at the actual ETC setting.

Figure 1 illustrates the VR model in action. The VR model includes an aerial view that allows the simulation master to explain the layout and site plan of the ETC and a first-person perspective that is controlled by using a "games controller." The trainee takes a virtual walk through the intricacies of the ETC while receiving immediate oversight training by experienced educators. The achievable learning objectives and the time needed to accomplish them are listed in Table 1.

CRIMEDIM is additionally capitalizing on the potentialities of the VR simulation model by running hybrid skills training exercises through its integration with mannequins, task-trainers, and actual equipment. The hybrid approach can effectively raise the educational target level from a level of understanding to a level of training (Table 1). By immersion in a highly realistic environment through the visualization of the virtual ETC on a screen or on the wall, the trainees are asked to accomplish competency-based specific tasks and to hone precise operational skill sets by using real equipment and performing procedures on mannequins. These skill sets focus on the use of PPE, peripheral venous cannulation, isolated blood drawing, cleaning and bathing the patient, the removal of bodily remains, and the safe disposal of dead bodies. It is anticipated that by increasing the levels of simulation fidelity, this innovative combination of tools with procedures will lead to a positive impact on the learning outcomes by facilitating greater knowledge gain and procedural skill acquisition. Furthermore, the hybrid simulation model provides the trainees the opportunity to integrate different evaluation methodologies, such as web-based assessment tools customized to the protocols used, the use of fluorescent nontoxic liquid or powder that glows under ultraviolet light to measure the degree of exposure by photographing the amount of fluorescence present on the worker's skin, ${ }^{6}$ and video cameras for educational replay and educational analysis. These tools allow for valid delivery and detailed feedback immediately during and after the exercise by the definitive detection of small but critical breaches in protections and protocols that have led to safety violations and potential transmission of the virus. Lastly, this simulation-based training is also intended to deliver team training exercises and to run emergency case scenarios in order to develop transversal

\section{FIGURE 1}

Pilot Hybrid Simulation at a Save the Children International Pre-deployment Induction Training Program.
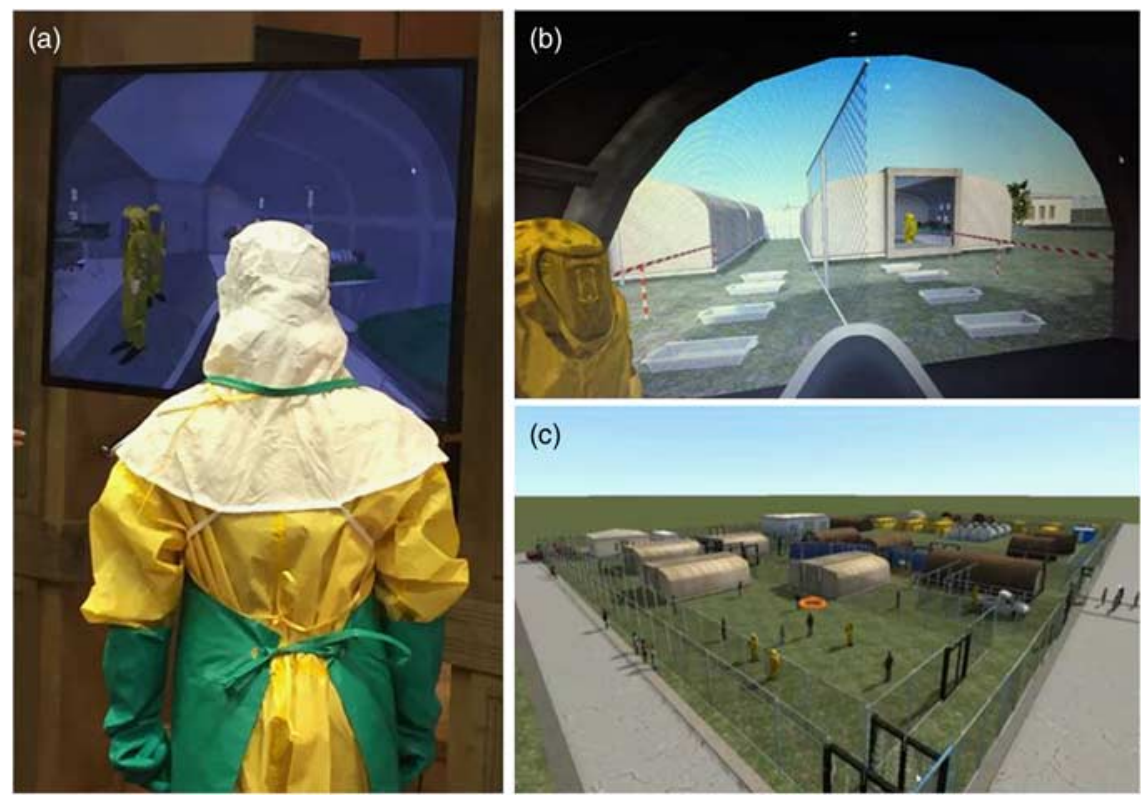

(a) Health care worker during a pre-deployment training simulation; (b) view of trainee walking through the virtualized ETC; (c) aerial view of the virtual reality version of the ETC. 
Example of a Set of Learning Objectives Achievable by Using the Virtual Reality Scenario and the Hybrid Simulation Model $^{\mathrm{a}}$

1. To describe the ETC

2. To recognize the different staff within the ETC

1. ETC Map

2. Staff involved

3. To explain the different zones inside the ETC

4. To describe the patient flow

5. To describe the staff flow

6. To recognize why PPE is needed according to the different areas

1. To demonstrate the PPE donning and doffing procedures

2. To demonstrate the skills required to use the protocols in place at the ETC setting

\author{
1. PPE donning and doffing procedures Hybrid simulation Training \\ 2. Patient admissions \\ 3. Bathing the patient \\ 4. Sharps management in high-risk area \\ 5. Peripheral venous cannulation \\ 6. Cleaning of body fluid spill \\ 7. Dead body preparation \\ 8. Disinfection of bed area \\ 9. Waste management in high-risk area \\ 10. Contingency hazards \\ 11. Response plan \\ 12. Accidental exposure protocol \\ 13. Incident reporting system
}

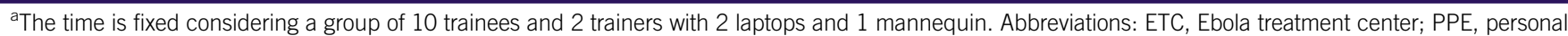
protective equipment.

skills, such as problem-solving, critical thinking, and the ability to work in teams. In detail, the VR scenario allows several trainees to walk around in the same virtual ETC; to communicate and cooperate, like they would in real life; and to analyze and contain the event and activate procedures.

A key benefit is that VR training is cost-effective and can be conducted in the field. In fact, the VR scenario requires only a common laptop, on which the XVR Software (E-Semble, Delft, the Netherlands) has been previously installed, along with a joypad to allow users to walk around in the simulated reality and to interact with the virtual objects. Both the software and the scenario are small files that can be easily transferred from one computer to another. A training unit to run the hybrid scenarios could be easily implemented in any room or in any tent equipped with a number of laptops equal to the number of trainees to train at the same time. Additionally, once a trainer has worked with the system, he or she can train others. The location flexibility of the training means that protocol can be easily standardized and coordinated across regions and repeated as many times as necessary without added expense.

\section{Limitations}

Limitations of the VR system have been identified. The hybrid model might be difficult in training a large group of people in a short period of time, because each trainee will need an individual computer station to ensure the effectiveness of the VR simulation. Moreover, the version of the XVR Software used to replicate the ETC has a library of virtual items with a finite number of objects and environments.

\section{CONCLUSIONS}

VR training is designed to increase staff safety and create a safe and realistic environment in which trainees can gain realistic basic and advanced skills. The goals are to accomplish complex task-performance behaviors, many of which carry life-or-death risks, and receive vital yet objective feedback before taking on the risk of working within the treatment center itself. While this is the first training of its kind, SCI plans to immediately roll out the training to staff in Sierra Leone, Liberia, and Guinea.

Additionally, hybrid simulation training incites an even more realistic and effective educational opportunity. Hybrid simulation training provides for "virtual exposure" to new operational public health skills essential for infection control and Ebola treatment management while reducing the exposure rate among health care providers. This awareness can instill confidence and greater willingness to engage in care delivery and strengthen operational public health skills for controlling and containing the outbreak.

\section{About the Authors}

CRIMEDIM - Research Center in Emergency and Disaster Medicine and Computer Science applied to Medical Practice, Università del Piemonte Orientale, Novara, Italy 
(Drs Ragazzoni, Ingrassia, Maccapani, Della Corte); Save the Children International, London, United Kingdom (Dr Echeverri and Ms Berryman); and Harvard Humanitarian Initiative, Harvard University, Cambridge, Massachusetts (Dr Burkle)

Correspondence and reprint requests to Luca Ragazzoni, CRIMEDIM - Research Center in Emergency and Disaster Medicine and Computer Science applied to Medical Practice, Università del Piemonte Orientale, via Lanino 1, 28100, Novara, Italy (e-mail: luca.ragazzoni@med.uniupo.it).

Published online: March 18, 2015.

\section{REFERENCES}

1. Errett NA, Barnett DJ, Thompson CB, et al. Assessment of medical reserve corps volunteers' emergency response willingness using a threatand efficacy-based model. Biosecur Bioterror. 2013;11:29-40.
2. Unprecedented number of medical staff infected with Ebola. Situation assessment, 25 August 2014. World Health Organization website. http://www. who.int/mediacentre/news/ebola/25-august-2014/en/. Accessed November 8, 2014.

3. Burkle FM. Operationalizing public health skills to resource poor settings: is this the Achilles heel in the Ebola epidemic campaign? Disaster Med Public Health Prep. 2014;7:1-3.

4. McGaghie WC, Issenberg SB, Petrusa ER, et al. A critical review of simulationbased medical education research: 2003-2009. Med Educ. 2010;44:50-63.

5. Ingrassia PL, Ragazzoni L, Carenzo L, et al. Virtual reality and live simulation: a comparison between two simulation tools for assessing mass casualty triage skills. Eur J Emerg Med. 2014; May 23 [Epub ahead of print].

6. Spray germ challenge: help make $\mathrm{HCW}$ safety a viral issue! CBRNE Collaborative For Health Care website. http://www.cbrnecc.ca/pandemicorange. Accessed February 18, 2015. 